\title{
Os limites da Escola Pública no enfrentamento da obesidade infantil
}

\section{The limits of Public School in fighting childhood obesity}

\author{
${ }^{1}$ Natália Rodrigues Moreira
}

Artigo

${ }^{2}$ Alden dos Santos Neves

Original

\begin{abstract}
Palavras-chave
Obesidade infantil

Escola pública

Promoção de saúde na escola

\section{Resumo}

Segundo estudiosos, a obesidade é definida como um excesso de gordura corporal relacionado à massa magra e está cada vez mais prevalente em crianças, o que se torna ainda mais preocupante. Esta pesquisa objetiva realizar uma investigação sobre os limites que as escolas encontram para combater a obesidade infantil e transmitir para os discentes hábitos mais saudáveis de alimentação. Esta análise partiu da necessidade de se verificar quais as limitações encontradas pelas escolas públicas, para mudar os hábitos alimentares dos alunos e ensiná-los a obterem hábitos alimentares saudáveis. Para tanto, foi proposto uma pesquisa interpretativa, com base na análise documental e de conteúdos com a finalidade de mostrar os limites das escolas públicas para enfrentar a obesidade infantil, e a importância do nutricionista trabalhar juntamente com.
\end{abstract}

\section{Abstract}

According to scholars obesity is defined as an excess of body fat related to lean mass and is increasingly prevalent in children, what is even more worrying. This research has objective to conduct an investigation into the limits that schools are to combat childhood obesity and transmit to the students more healthy eating habits. This analysis was based on the need to verify what the limitations faced by public schools, to change the eating habits of students and teach them how to achieve healthy eating habits. Therefore it was proposed interpretive research, based on analysis of documents and content in order to show the limits of public schools to address childhood obesity and the importance of working together nutritionist with the school community. It can be concluded that training is required for teachers, the inclusion of a healthy.
Keywords

Childhood obesity

Public school

School health

promotion 


\section{Introdução}

Durante as últimas décadas, a obesidade vem aumentando significativamente. Anos atrás, ela afetava, em sua maioria, apenas a adultos, porém, nos dias atuais, esse problema não atinge apenas os adultos, mas também ,crianças na idade escolar. Um dos fatores são as mudanças do estilo de vida da população, inclusive nos hábitos alimentares. A inversão do estado nutricional dos brasileiros da desnutrição para a obesidade mostra um importante aumento dos casos de sobrepeso e obesidade especialmente em crianças na idade escolar e em adolescentes (FERNANDES et al. 2009; SILVA et al., 2009).

As crianças obesas possuem maior chance de desenvolver precocemente diversas doenças como diabetes mellitus, hipertensão arterial, doenças cardiovasculares, problemas sociais e psicológicos. Além dessas doenças, ainda podem ter a permanência dessa obesidade na idade adulta (FILGUEIRAS et al., 2012).

O crescimento da obesidade e de dislipidemias em crianças mostram a necessidade de atenção e de implementação de medidas intervencionistas no combate e prevenção desse distúrbio nutricional, juntamente com os familiares, profissionais da área da saúde e também da área de educação, sabendo-se que a escola exerce grande influência na vida dos seus alunos, transmitindo valores que são expressos na escola por meio de aspectos concretos como a qualidade da merenda escolar (SILVA et al., 2010).

Para isso é de grande importância o conhecimento sobre nutrição por todos os profissionais que atuam direta ou indiretamente no campo da alimentação infantil, para a melhoria do estado nutricional das crianças, pois, além de contribuírem na formação do hábito alimentar destas, podem auxiliar os pais a fim de diminuir ou até mesmo evitar a obesidade infantil (SILVA et al., 2010).

Cada aluno traz para a escola, além de seu conhecimento de mundo, hábitos alimentares, sociais e culturais, esses são transmitidos pelos próprios familiares e sustentados por tradições. Com isso, nota-se que, os hábitos alimentares dos pais estão diretamente relacionados ao desenvolvimento do comportamento alimentar dos filhos. Porém, o comportamento alimentar é mutável, isto é, pode-se modificar-se ao longo da vida (MIRANDA; NAVARRO, 2008).

Segundo Bernandon e colaboradores (2009), em uma capacitação realizada em quatro escolas do Distrito Federal, educadores participantes relataram, que, apesar de reconhecerem os hábitos errôneos dos alunos e saberem sobre a importância da educação nutricional, muitas vezes, os deixam praticar a alimentação não conveniente. Isso pode acontecer, talvez, por faltar, nos docentes certos conhecimentos sobre alimentação saudável, fazendo com que estes não interfiram na formação dos hábitos alimentares das crianças.

Logo, nota-se que a necessidade de uma capacitação para os educadores a fim de que estes saibam explicar aos alunos sobre a importância de se ter hábitos alimentares saudáveis. Alguns docentes acreditam que não interferem nos hábitos alimentares de seus alunos, por falta de tempo para ações educativas sobre esse assunto, sendo necessária a criação de uma nova disciplina (BERNARDON et al., 2009).

Muitos alimentos encontrados em cantinas escolares, os quais os estudantes têm preferência, são muito energéticos, calóricos, ricos em açúcares, gorduras e sal, podendo causar a obesidade. As cantinas e os refeitórios devem ser espaços que estimulem e reforcem os hábitos alimentares saudáveis, e os docentes, em salas de aula, podem e devem abordar esses temas a fim de mudar essa realidade tão comum nos dias atuais (SCHMITZ et al., 2008).

A escola pode trabalhar para prevenir e reduzir o sobrepeso e obesidade na população infantil, bem como auxiliar no tratamento destes. Nota-se que em escolas onde não há cantina e a merenda escolar a é servida pela própria instituição há mais possibilidade de contribuição para a inserção de hábitos alimentares mais saudáveis (NETO et al., 2010).

Diante do exposto, este artigo objetiva realizar uma investigação sobre os limites que as escolas encontram para combater a obesidade infantil.

\section{Metodologia}

Esta pesquisa é uma revisão bibliográfica descritiva, realizada de setembro de 2011 a outubro de 2012, através da busca de artigos 
científicos, sites (IBGE e FNDE), monografias e resoluções, publicados entre 2006 e 2012, nas bases de dados eletrônicos (Scielo, Lilac e Biblioteca Virtual em Saúde).

\section{Desenvolvimento}

\subsection{Obesidade e sobrepeso no Brasil}

Entre vários problemas de saúde pública no Brasil, um dos que mais vem se destacando é a obesidade e o sobrepeso, esses são encontrados em todas as regiões brasileiras. Pode-se considerar que o problema tornou-se uma epidemia mundial. Considera-se o sobrepeso como uma proporção relativa de peso maior que a desejável para a altura (FLORENTINO; FERREIRA, 2011).

De acordo com Filgueiras e colaboradores (2012), “... a obesidade é uma doença crônica caracterizada pelo excesso de massa adiposa em relação ao peso corporal total que resulta em efeitos deletérios para a saúde".

A obesidade em crianças e adolescentes tem crescido em grande parte dos países, indicando um dos mais importantes problemas nutricionais atualmente; vem se tornando frequente até mesmo em países em desenvolvimento,onde pode-se presenciar casos tanto de desnutrição como de obesidade em uma mesma casa (LOPES et al., 2010).

\subsection{Obesidade e sobrepeso infantil}

A sociedade contemporânea está vivendo a transição nutricional da desnutrição para a obesidade. Por isso, se faz necessário o acompanhamento nutricional das crianças desde cedo. Fatores sociais, econômicos e culturais podem determinar o aumento da prevalência da obesidade, a qual vem sendo observada também em cidadãos das classes sociais mais baixas, onde estes podem obter sérios problemas de doenças crônico-degenerativas (NETO et al., 2010).

De acordo com a Organização Mundial de Saúde (OMS) e Organização Pan-Americana de Saúde (OPAS) os casos de obesidade infantil aumentaram muito e se torna cada vez mais preocupante a situação. No mundo, existem 17,6 milhões de crianças obesas com idade inferior a cinco anos. Desde a década de 60, o número de crianças obesas entre 6 e 11 anos, dobrou. Trata-se de um problema global que atinge os países desenvolvidos de forma crescente e é responsável por 2 a $6 \%$ do custo total de atenção à saúde (LOPES et al., 2010).

Segundo dados da Pesquisa de Orçamentos Familiares (POF), o número de crianças de 5 a 9 anos com excesso de peso teve um elevado salto ao longo de 34 anos. Em 2008-09, 34,8\% dos meninos estavam com o peso acima da faixa considerada saudável pela OMS. Em 1989, esse índice era de $15 \%$, contra $10,9 \%$ em 1974 75. Observou-se um padrão semelhante nas meninas, que de $8,6 \%$ na década de 70 foram para $11,9 \%$ no final dos anos 80 e chegaram aos $32 \%$ em 2008 -09 (IBGE, 2008 - 2009).

Os errôneos hábitos alimentares, os quais se caracterizam pelo aumento no consumo de alimentos altamente calóricos, principalmente os industrializados, juntamente com a diminuição da atividade física, resulta em um desequilíbrio energético, sendo esses os principais fatores que auxiliam no aumento da prevalência da obesidade e são influenciados diretamente pelos hábitos dos pais (MIRANDA; NAVARRO, 2008; SIMON et al., 2009).

Uma alimentação inadequada, além de causar a obesidade, pode resultar em alterações do aprendizado e da atenção, e também pode ocasionar problemas psicossociais, levando a criança ou adolescente a ter baixa autoestima e podendo levar ao isolamento social (GRANADA; GOLDSMITCH, 2008; MIRANDA; NAVARRO, 2008).

Para que se estabeleça um tratamento da obesidade infantil é necessária uma avaliação precoce e eficaz, desenvolvendo programas apropriados, juntamente com uma equipe formada por nutricionista, pediatra, psicólogo e educador físico, incluindo alterações nos hábitos alimentares e no estilo de vida físico (MIRANDA; NAVARRO, 2008).

\subsection{Os limites apresentados nas escolas públicas para o ensino-aprendizagem de hábitos alimentares saudáveis}

Com o crescimento descontrolado da obesidade e do sobrepeso infantil, os problemas relacionados à obesidade passaram a ser não somente de responsabilidade dos funcionários da área da saúde, mas sim de todos que trabalham 
diretamente ou indiretamente com crianças e adolescentes (SILVA et al., 2010).

As instituições escolares são espaços privilegiados para a promoção da saúde e desempenham papel fundamental na formação de hábitos, valores e estilos de vida, inclusive o da alimentação, em função dessas crianças e adolescentes passarem boa parte das horas de seus dias inseridos nesses locais. A alimentação escolar propicia a socialização de seus discentes favorecendo sua interação e aprendizagem (GRANADA; GOLDSMITCH, 2008).

A alimentação escolar no Brasil surgiu na década de 30 como um meio de correção nutricional em crianças de classes mais baixas as quais eram sujeitas aos diversos tipos e graus da desnutrição. Atualmente, ela está inscrita na Constituição Federal Brasileira como um direito de todas as crianças e adolescentes que frequentam, desde a Educação Infantil até o $9^{\circ}$ ano do Ensino Fundamental (GRANADA; GOLDSMITCH, 2008).

A educação alimentar é conteúdo previsto no Parâmetro Curricular Nacional (PCN), devendo ser trabalhada na aula de ciências, visando à melhoria do estilo de vida, por meio de escolhas alimentares saudáveis. Mas, para que o professor se transforme em agente promotor de hábitos alimentares saudáveis, é importante que possua, além do conhecimento teórico de dieta equilibrada, uma postura consciente de sua atuação na formação dos hábitos alimentares das crianças (FONTES, 2011).

A educação nutricional deveria ser obrigatoriamente incluída no currículo escolar, propondo atividades que possibilitem aos alunos a reflexão e a busca de estratégias para pequenas mudanças em seu cotidiano, modificando seu estilo de vida e a qualidade de sua alimentação, visando à saúde e a qualidade de vida. (MIRANDA; NAVARRO, 2008).

Porém, como os docentes devem cumprir seus planejamentos e essa disciplina não está inserida no currículo das escolas, muitos professores não trabalham de forma aprofundada o tema (BERNARDON et al., 2009).

Uma das causas do aumento na prevalência de obesidade e sobrepeso entre esses indivíduos é o tipo de alimentação. O nutricionista e a comunidade escolar devem trabalhar juntos para a conscientização e planejamento da alimentação saudável. Porém, nas escolas, há a presença de algumas barreiras, como por exemplo, o lanche escolar, este pode ser um obstáculo à alimentação adequada, tanto os disponíveis nas cantinas quanto os preparados em casa, e os distribuídos na merenda escolar, pois, a preferência dos indivíduos na idade infantil ou na adolescência é por alimentos gordurosos, calóricos, com grande teor de sal ou de açúcar, entre outros (SCHMITZ et al., 2008; NETO et al., 2010).

Programas de reeducação alimentar e a avaliação nutricional deveriam ser fortes aliados contra a obesidade em instituições escolares, consultórios e serviços públicos de saúde, tentando controlar os problemas de excesso de peso, principalmente em idades como a dos pré-escolares (SIMON et al., 2009).

É importante que, antes dos dez anos de idade ou na adolescência, sejam introduzidos hábitos alimentares saudáveis, podendo-se reduzir a gravidade de doenças, de forma mais eficaz do que se esses hábitos forem introduzidos na idade adulta (CARVALHO et al., 2009).

De acordo com o Programa Nacional de Alimentação Escolar (PNAE), a merenda escolar tem a finalidade de atender às necessidades nutricionais dos alunos durante o período que estão na escola e também promover hábitos alimentares saudáveis (CARVALHO et al., 2010).

O cardápio ideal escolar seria uma merenda de composição variada com aproximadamente $450 \mathrm{kcal}$, tendo sempre produtos de qualidade e cuidados que devem ir desde a compra até a distribuição, e preparados dentro das técnicas de higiene (CARVALHO et al., 2009).

As cantinas escolares devem adotar um cardápio com alimentos menos calóricos e mais nutritivos e saudáveis, não basta apenas fornecer alimentos aos alunos, é fundamental que essa alimentação fornecida seja equilibrada para recuperar e manter o estado nutricional adequado (FREITAS, 2010; PONTES et al., 2011). Os programas de educação nutricional devem ir além das atividades em sala de aula. O objetivo da merenda escolar é complementar as necessidades nutricionais da criança, porém, em casos de extrema pobreza essa "complementação" pode ser a única refeição do dia (GRANADA; GOLDSMITCH, 2008).

Como uma política pública essencial às necessidades nutricionais, de saúde e sociais 
da população escolar, faz-se obrigatória a implementação da educação nutricional desde o ensino fundamental, propondo a construção coletiva do conhecimento, mediante planejamento participativo com integração entre a equipe de saúde, a escola, a criança e a família, pois, na idade escolar, um trabalho realizado em grupo, nas escolas é de grande importância (MIRANDA; NAVARRO 2008).

Como parte das políticas públicas para combate à obesidade, com base no ambiente escolar, foi promulgada em 08 de maio de 2006, a Portaria Interministerial, $n^{\circ}$. 1.010. Suas diretrizes baseiam-se nas ações de educação alimentar e nutricional, estímulo à produção de hortas escolares, implantação de boas práticas de manipulação, monitoramento da situação nutricional e regulamentação do comércio de alimentos: restrição ao comércio no ambiente escolar de alimentos e preparações com altos teores de gordura saturada, gordura trans, açúcar livre e sal, com incentivo ao consumo de frutas e hortaliças.

A fim de construir políticas públicas intersetoriais, o governo Federal criou o Programa Saúde na Escola (PSE), lançado pelo Ministério da Saúde e da Educação, através do Decreto $n^{0}$ 6.286, de 5 de dezembro de 2007, objetivando contribuir para a formação integral dos estudantes da rede pública de educação básica por meio de ações de prevenção, promoção e atenção à saúde, visando á melhoria da qualidade de vida da população brasileira. O PSE beneficia estudantes da Educação Básica, gestores e profissionais de saúde e educação, comunidade escolar e também estudantes da Rede Federal de Educação Profissional e Tecnológica e da Educação de Jovens e Adultos (EJA). Essa iniciativa reconhece e acolhe as ações de integração entre saúde e educação que já existem e que tem colaborado positivamente na qualidade de vida dos estudantes. Dentre as atividades previstas, está a avaliação nutricional; prevenção precoce de hipertensão e diabetes; saúde bucal; acuidade visual e auditiva; avaliação psicológica; prevenção e promoção da saúde - o que irá trabalhar com a construção da cultura de paz, combate a violência, álcool e outras drogas como tabaco; educação sexual e reprodutiva; estímulo à atividade física; educação permanente; capacitação de profissionais e de jovens; e, monitoramento e avaliação da saúde dos estudantes (PORTAL DA SAÚDE; PORTAL BRASIL, 2012).

Devido a necessidade no Brasil e no mundo de mudanças nos perfis nutricionais, foi necessário implementar estratégias de promoção da alimentação saudável para escolares. Santa Catarina foi o primeiro estado brasileiro a criar uma legislação específica (lei estadual 12.061/2001) para regulamentar os alimentos comercializados nas cantinas escolares. Alguns estados como Paraná, Mato Grosso, São Paulo e Rio Grande do Sul adotaram a ideia e vem regulamentando esse comércio nas escolas. No Distrito Federal, para a promoção da alimentação saudável nas instituições escolares, criou-se um projeto de lei em que se registra que a cantina deve ser administrada por pessoas capacitadas em aspectos de alimentação e nutrição, relevantes para o exercício do comércio de alimentos destinados as crianças e jovens (REIS et al., 2011).

\subsection{A importância do nutricionista nas escolas públicas}

Vários são os projetos que o governo federal tem estabelecido para promover a saúde alimentar nas escolas públicas, entre eles tem- se o PNAE que, ao longo do tempo, vem sofrendo alterações benéficas para a alimentação escolar. Através de equipes de Nutrição, diretrizes, resoluções e leis vem-se capacitando o Nutricionista Escolar nas atribuições de sua função (FNDE, 2012).

Com base nessa política de alimentação, observa-se o avanço e consolidação no âmbito escolar público. O importante é que essas políticas estabelecidas contam também com o apoio e supervisão da sociedade (conselhos gestores), como exemplo, na Alimentação Escolar existe o CAE (Conselho da Alimentação Escolar) em que na sua composição participam membros da sociedade civil, pais de alunos, professores e outros. Outro exemplo é a aquisição de gêneros alimentícios vindos da agricultura familiar (FNDE, 2012).

O nutricionista é o profissional capacitado para promover a saúde na escola, através de atividades educativas e assistenciais que enfatizem hábitos alimentares saudáveis e promovem o envolvimento familiar (MIRANDA; NAVARRO, 2008). 
O profissional de nutrição no PNAE tem o objetivo de monitorar o estado nutricional dos estudantes; programar, elaborar e avaliar os cardápios da alimentação escolar, levando em consideração os perfis epidemiológicos, as idades dos alunos atendidos, a cultura alimentar de cada local e a vocação agrícola da região; acompanhar, desde a aquisição dos gêneros alimentícios até a produção e distribuição da alimentação e realizar ações de educação alimentar e nutricional nas instituições escolares, de modo que promova hábitos alimentares saudáveis (SANTOS et al., 2012).

A inserção do nutricionista nas instituições educacionais como, por exemplo,em creches, escolas e nos meios de comunicação, também devem ser estimuladas a fim de fortalecer as ações de educação nutricional (MIRANDA; NAVARRO, 2008).

\section{Considerações Finais}

Conclui-se que as instituições escolares são locais propícios para o desenvolvimento de ações educativas em saúde e demonstra a gran- de necessidade de implantações de programas para a prevenção da obesidade infantil com o intuito de formar hábitos alimentares adequados e influenciar um estilo de vida saudável, devido ao grande tempo que os alunos passam inserido nesses lugares. As políticas públicas existentes são fundamentais para garantia do direito a alimentação adequada e para obter mudança nos quadros de sobrepeso e obesidade.

Os principais limites, apresentados na atualidade, para a utilização da escola enquanto espaço promotor de saúde, foi a merenda levada pelo estudante, a falta de capacidade dos professores em ensinar sobre alimentação saudável aos alunos e falta do nutricionista nestas instituições.

Para isso, verifica-se a necessidade de uma capacitação para os docentes, a inserção do tema alimentação saudável como conteúdo educativo, a criação de uma nova disciplina de educação nutricional, a adequação das merendas escolares e a presença do nutricionista na rede pública escolar a fim de preparar o cardápio dos alunos, acompanhar o momento das refeições e fazer um acompanhamento direto e contínuo com professores, alunos, pais e familiares.

\section{Referências Bibliográficas}

BERNARDON, R.; SILVA, J. R. M. da.; CARDOSO, G. T.; MONTEIRO, R. A.; AMORIM, N. F. de A.; SCHMITZ, B. de A. S.; RODRIGUES, M. de L. C. F. Construção de metodologia de capacitação em alimentação e nutrição para educadores. Revista de Nutrição, v. 22, n. 3, p. 389 398, 2009.

BRASIL. Portaria Interministerial $n^{\circ} 1.010$ de 8 de maio de 2006. Institui as diretrizes para a Promoção da Alimentação Saudável nas Escolas de educação infantil, fundamental e nível médio das redes públicas e privadas, em âmbito nacional. Diário Oficial da União 2006. Disponível em: $<$ http://dtr2001.saude.gov.br/sas/PORTARIAS/Port2006/GM/GM-1010.htm>. Acesso em: 20 de outubro de 2011.

CARVALHO, A. P.; OLIVEIRA, V. B.; SANTOS, L. C. Hábitos alimentares e práticas de educação nutricional: atenção a crianças de uma escola municipal de Belo Horizonte, Minas Gerais. Pediatria, v. 32, n. 1, p. 20 - 27, 2010.

CARVAlHO, M. F. de.; CARVALHO, R. F. de.; CRUZ, F. L. G.; RODRIGUES, P. A.; LEITE, F. P. P.; CHAVES, M. das G. A. M. Correlação entre a merenda escolar, obesidade e cariogenicidade em escolares. Revista Odonto, v. 17, n. 34, p. 56 - 63, 2009. 
FERNANDES, P. S.; BERNARDO, C. de O.; CAMPOS, R. M. M. B.; VASCONCELOS, F. de A. G. Evaluating the effect of nutritional education on the prevalence of overweight/obesity and on foods eaten at primary schools. Jornal de Pediatria, v. 85, n. 4, p. 315 - 321, 2009.

FILGUEIRAS, M. de C.; RIPARDO, N. V. de L.; SOUZA, S. dos S.; MOREIRA, A. K. de F. Prevalência de obesidade em crianças de escolas públicas. Revista Ciência \& Saúde, v. 5, n. 1, p. $4-47,2012$.

FLORENTINO, C. B.; FERREIRA, C. C. D. A influência de assistir televisão na prevalência de sobrepeso e obesidade em crianças e adolescentes. 18f. Trabalho Monográfico (Graduação em Nutrição), Centro Universitário de Volta Redonda - UniFOA, 2011.

FONTES, P. G. O professor como influenciador de hábitos alimentares saudáveis na escola. $25 f$. Trabalho Monográfico (Graduação em Ciências Naturais), Universidade de Brasília - Faculdade UnB Planaltina, 2011.

FREITAS, H. R. M. Análise da prevalência de obesidade em escolares da rede de ensino particular de Morada Nova. 60f. Trabalho Monográfico (Graduação em Educação Física), Faculdade Católica Rainha do Sertão, 2010.

Fundo Nacional de Desenvolvimento da Educação. O PNAE: desafios e Oportunidades. Disponível

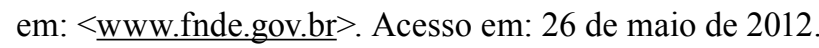

GRANADA, G. G.; GOLDSCHMIDT, P. da S. Biodisponibilidade de ferro na merenda escolar. Alimentos e Nutrição, v. 19, n. 4, p. 441 - 448, 2008.

Instituto Brasileiro de Geografia e Estatística (IBGE). Pesquisa de Orçamentos Familiares. 2008-2009

LOPESP. C. S.; PRADO, S. R. L. de A.; COLOMBO, P. Fatores de risco associados à obesidade e sobrepeso em crianças em idade escolar. Revista Brasileira de Enfermagem, v. 63, n. 1, p. 1 - 7, 2010.

MIRANDA, A. A. N. de.; NAVARRO, F. Prevenção e o tratamento da obesidade durante a infância: Uma opção eficaz para reduzir a prevalência desta patologia. Revista Brasileira de Obesidade, Nutrição e Emagrecimento, v. 2, n. 10, p. 313 -323, 2008.

NETO, J. O.; OLBRICH, S. R. L. R.; MORI, N. L. R.; SOARES, P. O.; CORREA, B. M. C. Avaliação nutricional em crianças de uma instituição de ensino. A escola que alimenta? Revista Ciência em Extensão, v. 6, n. 1, p. 1 - 12, 2010.

PONTES, R.; SLOMPO, R. B.; LUZ, P. A. da.; PASSONI, C. M. S. Influência da merenda escolar no estado nutricional. Cadernos da Escola de Saúde, v. 1, n. 4, p. 64 - 77, 2011.

PORTAL BRASIL - Programa Saúde na Escola. Disponível em: < http://portalsaude.saude.gov.br/ portalsaude/area/7/o-ministerio.html $>$ Acesso em 24 de novembro de 2012.

PORTAL BRASIL. Saúde na Escola. Disponível em: < http://www.brasil.gov.br/sobre/cidadania/ brasil-sem-miseria/acesso-a-servicos/saude-na-escola/print $>$ Acesso em: 24 de novembro de 2012.

REIS, C. E. G.; VASCONCELOS, I. A.; BARROS, J. F de N. Políticas públicas de nutrição para o controle da obesidade infantil. Revista Paulista de Pediatria, v. 29, n. 4, p. 625 - 633, 2011. 
SANTOS, L. A. da S.; PAIVA, J. B. de.; MELLO, A.; FONTES, G. A. V.; SAMPAIO, L. R.; FREITAS, M. do C. S. de. O nutricionista no programa de alimentação escolar: avaliação de uma experiência deformação a partir de grupos focais. Revista de Nutrição, v. 25, n. 1, p. 107 - 117, 2012.

SCHMITZ B. de A. S.; RECINE, E.; CARDOSO, G. T.; SILVA, J. R. M. da.; AMORIM, N. F. de A.; BERNARDON, R.; RODRIGUES, M. de L. C. F. A escola promovendo hábitos alimentares saudáveis: Uma proposta metodológica de capacitação para educadores e donos de cantina escolar. Caderno de Saúde Pública, v. 24, n. 2, p. 312 - 322, 2008.

SILVA, A. C. de A.; JÚNIOR, R. T.; MONTEIRO, M. I. Analisando conhecimentos e práticas de agentes educacionais e professoras relacionados à alimentação infantil. Ciência \& Educação, v. 16, n. 1, p. 199 - 214, 2010.

SILVA, A. R. V. da.; DAMASCENO, M. M.C.; MARINHO, N. B. P.; ALMEIDA, L. S. de.; ARAÚJO, M. F. M. de.; ALMEIDA, P. C.; ALMEIDA, I. S. de. Hábitos alimentares de adolescentes de escolas públicas de Fortaleza, CE, Brasil. Revista Brasileira de Enfermagem, v. 62, n. 1, p. $1-8,2009$.

SIMON, V. G. N.; SOUZA, J. M. P. de; LEONE, C.; SOUZA S. B. de. Prevalência de sobrepeso e obesidade em crianças de dois a seis anos matriculadas em escolas particulares no município de São Paulo. Revista Brasileira de Crescimento Desenvolvimento Humano, v. 19, n. 2, p. 211 - 218, 2009. 